\title{
Fundamental Limits on Sensing Chemical Concentrations with Linear Biochemical Networks
}

\author{
Christopher C. Govern and Pieter Rein ten Wolde \\ FOM Institute AMOLF, Science Park 104, 1098 XG Amsterdam, Netherlands
}

(Received 27 June 2012; published 20 November 2012)

\begin{abstract}
Living cells often need to extract information from biochemical signals that are noisy. We study how accurately cells can measure chemical concentrations with signaling networks that are linear. For stationary signals of long duration, they can reach, but not beat, the Berg-Purcell limit, which relies on uniformly averaging in time the fluctuations in the input signal. For short times or nonstationary signals, however, they can beat the Berg-Purcell limit, by nonuniformly time averaging the input. We derive the optimal weighting function for time averaging and use it to provide the fundamental limit of measuring chemical concentrations with linear signaling networks.
\end{abstract}

Cells measure concentrations of chemicals via receptors on their surface. These measurements, however, are inevitably corrupted by noise that arises from the stochastic arrival of ligand molecules at the receptor by diffusion and from the stochastic binding of the ligand to the receptor. Biochemical networks that transmit the information on the ligand concentration from the surface of the cell to its interior often have to filter this noise extrinsic to the cell as much as possible. However, how the capacity of signaling networks to remove extrinsic noise depends on their design, and what the fundamental limits to this capacity are, remain open questions.

Several studies have addressed the question of how accurately the ligand concentration $c$ can be estimated from the time trace of the number of ligand-bound receptors, $S(t)$, over some integration time $T$ [1-8]. Berg and Purcell assumed that the estimate $\hat{c}$ with least error is the one that matches the observed time average of the stochastic signal $S(t), \bar{S}=1 / T \int_{0}^{T} S(t) d t$, giving all the signal values equal weight in the average [1]. When $S(t)$ is stationary, with mean $\mu_{S}$, variance $\sigma_{S}^{2}$, and correlations that decay exponentially over a time $\tau_{c}$, the estimate $\hat{c}$ has variance (error) $[3,9,10]$ :

$$
\sigma_{\hat{c}}^{2}[\bar{S}]=\frac{\sigma_{\bar{S}}^{2}}{\left(d \mu_{S} / d c\right)^{2}}=\frac{\sigma_{S}^{2} /\left(d \mu_{S} / d c\right)^{2}}{\frac{T}{2 \tau_{c}}\left(1-\frac{1-\exp \left(-T / \tau_{c}\right)}{T / \tau_{c}}\right)^{-1}} .
$$

More recently, Mora, Endres, and Wingreen showed that, when $T \gg \tau_{c}$, maximum likelihood estimation produces an estimate that is better by $50 \%$, since the time average includes noise from stochastic ligand unbinding, which provides no information about the ligand concentration $[5,8]$.

While these previous studies have considered how much information about the ligand concentration is encoded in receptor-occupancy time traces, they do not address the question of how much information biochemical networks can actually extract from these time traces. To extract all the information, the biochemical networks downstream of the receptors would need to construct a maximum likelihood estimate $[5,8]$. However, it is not clear that typical biochemical networks do this, nor is it clear that they time average signals uniformly as in the Berg-Purcell estimate. Moreover, the previous analyses [1-8] assumed an integration time $T$, but what time scales in the processing network actually set the integration time remains unclear. We therefore study how accurately biochemical networks can estimate the ligand concentration from receptor time traces.

We focus on a simple but broad class of signaling networks, linear networks [11]. Many networks respond linearly over the range of fluctuations in their input (e.g., Ref. [12-14]) and a systematic study can be done analytically. Since the effects of noise intrinsic to the molecular interactions inside cells have been well studied [14-17], we focus on networks in the deterministic limit. This enables us to understand the unique effects due to the noise in the input signal.

Linear networks time average the input signal, but do not generally give rise to uniformly weighted time averages. We study how different signaling motifs sculpt the weighting of the signal as a function of time, and how this affects the precision of ligand sensing. While linear networks cannot extract all of the information in the input signal (i.e., the maximum likelihood estimate [5]), they can, surprisingly, reach the Berg-Purcell limit and even exceed it by $12 \%$; this is because the optimal weighting is nonuniform, in contrast to the Berg-Purcell estimate. We show that a simple network based on a feed-forward loop, a common motif in biochemical networks [18], can reach the bound for linear signaling networks, and we elucidate the combination of time scales that sets the effective integration time. We conclude by studying how reliably biochemical signaling networks can extract information from nonstationary signals.

We consider a cell that responds after a finite time $T_{o}$ to a change in its environment which happens at time $t=0$. This time $T_{o}$ is the observation time, which, as we discuss below, provides an upper bound to the integration time. 
As before, the receptor time trace provides the signal to the cell, $S(t)$. To compare to previous results, we initially assume that the change in the environment, and therefore the ligand concentration, is instantaneous, and that the receptors immediately adjust. Moreover, we assume that the fluctuations in $S(t)$ decay exponentially with correlation time $\tau_{c}[19,20]$. We neglect stochasticity in the time $T_{o}$, and, as mentioned above, the intrinsic noise in the processing network. The capacity of the cell to respond is then limited by the information in the stationary input $S(t)$ from time 0 to $T_{o}$ [Fig. 1(a)].

As a measure of how much information the cell can extract, we determine how accurately the ligand concentration can be estimated from the molecular output $X$ of the processing network at the time $T_{o}$ of the response, assuming the response is made instantaneously based on $X\left(T_{o}\right)$. As illustrated below in examples, the output of a linear signaling network is $X\left(T_{o}\right)=\int_{-\infty}^{T_{o}} f\left(T_{o}-t^{\prime}\right) S\left(t^{\prime}\right) d t^{\prime}$, where the unnormalized weighting (response or transfer) function $f\left(\Delta t=T_{o}-t^{\prime}\right)$ reflects how the processing network at time $T_{o}$ weights the signal at an earlier time $t^{\prime}$ [21]. To compare to previous results, we assume that either: (1) $f\left(T_{o}-t\right)=0$ for $t<0$, which corresponds to a scenario where the response time $\tau_{r}$ of the network is shorter than $T_{o}$, or, equivalently, the network reaches steady state by the time $T_{o}$ [Fig. 1(b)]; or (2) $S(t)=0$ for $t<0$, which corresponds to a scenario in which the cell is initially in a basal state [Fig. 1(c)]. In both cases, we then have $X\left(T_{o}\right)=$ $\int_{0}^{T_{o}} f\left(T_{o}-t^{\prime}\right) S\left(t^{\prime}\right) d t^{\prime}$. When neither $f\left(T_{o}-t\right)$ nor $S(t)$ are zero for $t<0$, then previous states of the environment, corresponding to $t<0$, influence the state of the signaling network at time $T_{o}$. Such previous environmental states can be a source of additional noise in $X\left(T_{o}\right)$, complicating inference of the current environmental state, as well as a source of information, helping inference, if environmental transitions are correlated.
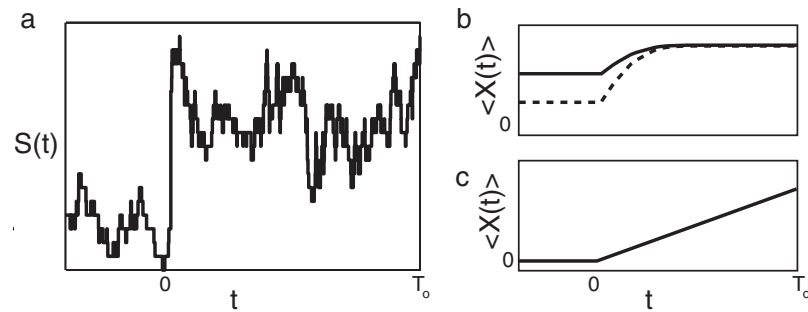

FIG. 1. Responding to noisy environments. (a) The environment changes instantaneously at time $t=0$, and the number of bound receptors, $S(t)$, adjusts instantaneously. $S(t)$ is stationary between time 0 and the time $T_{o}$, when the cell responds. The signaling network is either in a steady state by time $T_{o}$, independent of the initial condition (b), or in a basal state at time $t=0$ (c). $X(t)$ denotes the number of $X$ molecules at time $t$. The solid and dashed lines in panel (b) represent different initial conditions.
We start by considering a simple linear signaling network, a reversible one-level cascade, in which the output molecule $X$ is directly activated by the receptor with rate constant $k_{f}$ and can be degraded with rate constant $k_{b} \quad$ (Fig. 2(a)). Then, deterministically, $d X / d t=$ $k_{f} S-k_{b} X$. The response of this network at time $T_{o}$ is $X\left(T_{o}\right)=\int_{0}^{T_{o}} f\left(T_{o}-t\right) S(t) d t+g\left(T_{o}\right) X(0)$ with $f(\Delta t)=$ $k_{f} \exp \left(-k_{b} \Delta t\right)$ and $g\left(T_{o}\right)=\exp \left(-k_{b} T_{o}\right)$ (Fig. 2(a)). We neglect the term $g\left(T_{o}\right) X(0)$ for the reasons mentioned above: either because $T_{o}$ is larger than the response time $\tau_{r}=1 / k_{b}$ in which case $g\left(T_{o}\right) \approx 0$, or because the initial state is ligand-free and $X(0) \approx 0$. We note that the weighting function $f(\Delta t)$ decays with increasing $\Delta t$, which means that more weight is placed on more recent values of the input signal. This is because the decay reaction is least likely to have degraded the most recently produced $X$ molecules.

We now address the question how the departure from uniform weighting affects the error in the estimate of the concentration. Following the derivation of Eq. (1) [1], an estimate of the ligand concentration from $X\left(T_{o}\right)$ has variance

$$
\sigma_{\hat{c}}^{2}\left[X\left(T_{o}\right)\right]=\sigma_{X\left(T_{\mathrm{o}}\right)}^{2} /\left(d \mu_{X\left(T_{o}\right)} / d c\right)^{2},
$$

where the mean $\mu_{X\left(T_{o}\right)}$ of $X\left(T_{o}\right)$ is a linear function of $c$ over the range of fluctuations in $X\left(T_{o}\right)$. Using $X\left(T_{o}\right)=$ $\int_{0}^{T_{o}} f\left(T_{o}-t^{\prime}\right) S\left(t^{\prime}\right) d t^{\prime}$, we then arrive at [22]

$$
\begin{aligned}
\sigma_{\hat{c}}^{2} & {\left[X\left(T_{o}\right)\right] } \\
& =\sigma_{\hat{c}}^{2}[S] \int_{0}^{T_{o}} \int_{0}^{T_{o}} \bar{f}\left(T_{o}-t_{1}\right) \bar{C}\left(t_{1}, t_{2}\right) \bar{f}\left(T_{o}-t_{2}\right) d t_{1} d t_{2} .
\end{aligned}
$$

Here, $\sigma_{\hat{c}}^{2}[S]=\sigma_{S}^{2} /\left(d \mu_{S} / d c\right)^{2}$ is the error of an estimate based on an instantaneous observation of the signal $S(t)$. The reduction in error, resulting from averaging the fluctuations in the input signal over time, depends on the normalized correlation function of the input fluctuations, $\bar{C}\left(t_{1}, t_{2}\right)=\exp \left(-\left|t_{2}-t_{1}\right| / \tau_{c}\right)$, and on the normalized weighting function, $\bar{f}(\Delta t)=f(\Delta t) / \int_{0}^{T_{o}} f\left(\Delta t^{\prime}\right) d \Delta t^{\prime}$.

Figure 2(b) shows that the one-level reversible cascade extracts less information from the input signal than a network that averages the input uniformly over time. Only when $k_{b}$ goes to zero, and $f(\Delta t) \propto \exp \left(-k_{b} \Delta t\right) \approx 1$, does the network, which now becomes an irreversible one-level cascade, implement uniform time averaging and does it extract the same amount of information. Intuitively, degrading $X$ destroys information. While degradation is required to make a signaling network responsive to new environments, this example shows that it may be useful to make degradation as weak as possible or to physically separate the receptors and deactivating enzymes (e.g., in different domains on the membrane [23]), such that $X$ is deactivated only after the response has been made. 

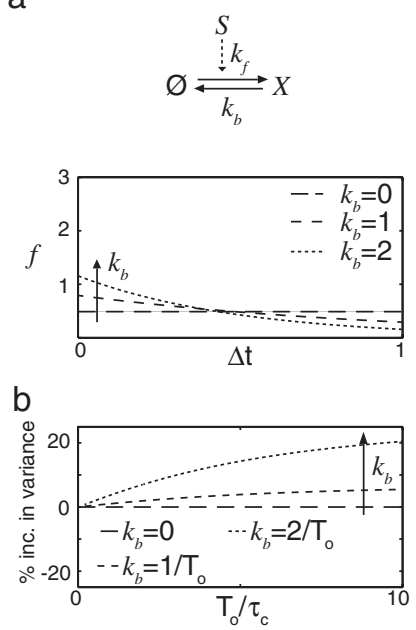

C

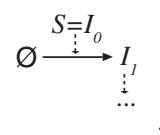

$\varnothing \stackrel{\cdots}{\longrightarrow} X=I_{N}$
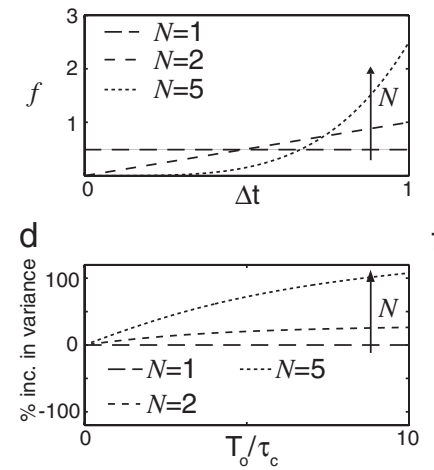

e

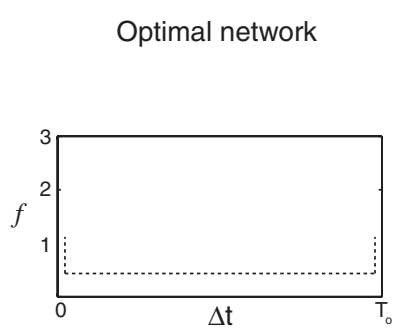

f

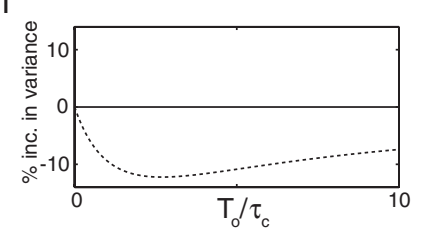

g
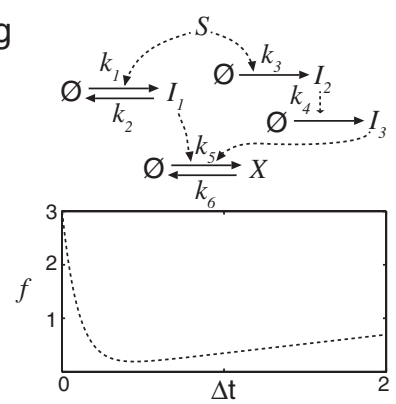

$\mathrm{h}$

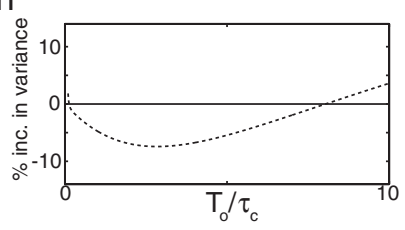

FIG. 2. Extracting information from noisy input signals with linear signaling networks. (a),(c),(e),(g) The weighting functions corresponding to different signaling networks are not uniform. (b),(d),(f),(h) The ability of a signaling network to measure ligand concentration depends on its weighting function. The typical error (variance) in the estimate of ligand concentration is plotted as a percentage increase over the error of an estimate based on uniform weighting, assumed in the Berg-Purcell limit [Eq. (1) with $T=T_{o}$ ]. (a) Reversible, one-level cascades selectively amplify late $\left(t=T_{o}\right)$ values of the signal, (b) leading to worse performance than the uniform average. (c) Irreversible, $N$-level cascades amplify early $(t=0)$ values of the signal, (d) leading to worse performance than the uniform average. (e) The optimal weighting function, given by Eq. (4), averages the signal, selectively amplifying less correlated values. The delta functions are truncated for illustration. (f) The optimal weighting function outperforms the uniform average. (g) A signaling network consisting of two branches, which selectively amplify late $\left(t=T_{o}\right)$ (left branch) and early $(t=0)($ right branch) values of the signal, approximates the optimal weighting function $\left(k_{1}=4.4 k_{3} k_{4} T_{o} ; k_{2}=20 / T_{o} ; k_{4}=0.35 k_{3} ; \bar{f}\right.$ independent of $\left.k_{3}, k_{5} ; k_{6} \gg T_{o}^{-1}\right)$. (h) The network in (f) can outperform the uniform average.

Signaling networks typically consist of more than one layer, which makes it possible to sculpt the weighting function $f(\Delta t)$. As an illustration, we first consider an irreversible cascade consisting of $N$ layers or species: $d I_{i} / d t=k_{f i} I_{i-1}$, where $i=1, \ldots, N \quad$ and $\quad I_{0}=S$. Assuming $X(0) \approx 0, X\left(T_{o}\right)=\int_{0}^{T_{o}} f\left(T_{o}-t\right) S(t) d t$, where the weighting function now behaves as $f(\Delta t) \propto \Delta t^{N-1}$. Such cascades place more weight on early values of the input signal, which have had more time to propagate through the network [Fig. 2(c)]. As a result, they underutilize (down-weight) the most recent information in the signal, and indeed, these cascades perform worse than a strict average of the input [Fig. 2(d)].

The above formalism can be generalized to arbitrarily large linear signaling networks. Multilevel reversible cascades have weighting functions that peak some finite time in the past, balancing the down-weighting of the signal from the distant past due to the reverse reactions, with the down-weighting of the signal from the recent past resulting from the multilevel character of the network [22]. More generally, linear combinations of the weighting functions for reversible and irreversible cascades can be achieved with multiple cascades that are activated by the input in parallel and which independently activate the same effector molecule, as we demonstrate below. Clearly, signaling networks allow for very diverse weighting functions.

This raises the question whether there exists an optimal weighting function $f^{*}(\Delta t)$ that minimizes the error in the estimate of the ligand concentration. To this end, we differentiate Eq. (3) with respect to $\bar{f}$ using Lagrange multipliers that constrain the integral of $\bar{f}$ to 1 , to find the optimal (normalized) weighting function:

$$
\bar{f}^{*}(\Delta t)=\left(1-w^{*}\right) \frac{1}{T_{o}}+w^{*} \frac{\delta(\Delta t)+\delta\left(\Delta t-T_{o}\right)}{2} .
$$

The first term places equal weight on all prior values of the input, as assumed in previous studies [1-4,7]. The second term, however, places greater weight on the first and last observed values of the signal, which are the two signal values that are the least correlated. Indeed, this is the central result of this manuscript: the optimal weighting function does not correspond to uniform weighting of all signal values. How much weight is placed on the first and last points is determined by $w^{*}=\frac{1}{T_{o} /\left(2 \tau_{c}\right)+1}$, which decreases from one to zero as the response time $T_{o}$ over the correlation time $\tau_{c}$ increases.

The optimal weighting function can be implemented using common network motifs. For example, the commonly observed feed-forward loop [18] in Fig. 2(g) contains two branches which independently activate $X$. The left branch, a one-level reversible cascade, amplifies later values of the signal $\left(t \rightarrow T_{o}\right)$; the right branch, a multilevel irreversible cascade, amplifies earlier $(t \rightarrow 0)$ values of the signal. Together, they produce a weighting function which selectively amplifies less correlated values of the input (Fig. 2(g) and 2(h)), outperforming the uniform average 
that could be obtained by reading out node $\mathrm{I}_{2}$ directly. This simple network illustrates how a spectrum of protein lifetimes and cascade levels can be used to shape weighting functions.

The optimal weighting function $f^{*}$ also provides the fundamental limit on the ability of linear signaling networks to measure chemical concentrations:

$$
\sigma_{\hat{c}}^{2}\left[X^{*}\left(T_{o}\right)\right]=\frac{\sigma_{\hat{c}}^{2}[S]}{T_{o} /\left(2 \tau_{c}\right)+1},
$$

which is obtained by combining Eqs. (3) and (4). Equation (5) has a simple interpretation: a time series of length $T_{o}$ contains an independent observation every time period of the order of the correlation time, plus one corresponding to the observation at $t=0$. Equation (5) is then the formula for the variance of the mean of $N=T_{o} /\left(2 \tau_{c}\right)+1$ independent, identically distributed random variables.

The improvement of the optimal weighting function over uniform weighting [Eq. (1)] is maximal when the observation time is about three correlation times. The maximum improvement over the sample average is $12 \%$ [Fig. 2(f)]. While this improvement over the Berg-Purcell estimate is modest, and smaller than the $50 \%$ improvement that could in principle be obtained by maximum likelihood $[5,8]$, it does show, for the first time, that simple signaling networks can indeed reach the Berg-Purcell limit, and even exceed it.

Equally important, our analysis provides a clear perspective on the integration time. Clearly, $T_{o}$, the time on which the cell must respond, provides an upper bound on the integration time. Yet, the processing network weights the input signal by $f\left(T_{o}-t\right)$, which may become zero for $t<T_{o}$. In this case, the effective integration time $T_{\text {eff }}$ is limited by the range over which $f\left(T_{o}-t\right)$ is nonzero. For example, the weighting function of the one-level reversible cascade becomes zero on the time scale $k_{b}^{-1}=\tau_{X}$, the lifetime of the output component. This can be (much) smaller than $T_{o}$, in which case $T_{\text {eff }}$ is limited by $\tau_{X}: T_{\text {eff }} \sim$ $\tau_{X}<T_{o}$. Essentially, degradation of the output erases memory of the input. However, our study of multi-level reversible cascades shows that in general the range over which $f(\Delta t)$ is non-zero can be longer than the lifetime of the individual components. Additional intermediate layers not only change the form of $f(\Delta t)$, but also extend the range over which it is nonzero, increasing the integration time over which the output remembers past signals [22].

Values for the correlation time $\tau_{c}$ of the input signal and the observation time $T_{o}$ vary widely across biological systems. Ligand-receptor half-lives, a key determinant of $\tau_{c}$, vary at least over more than an order of magnitude, i.e. from milliseconds to an hour $[6,24]$. The cell-cycle time provides an upper bound on $T_{o}$ [25] (e.g., $45 \mathrm{~min}$ in E. coli [25] and $100 \mathrm{~min}$ in yeast [26]), but signaling modules and transcriptional responses can make decisions sooner. Indeed, $T_{o}$ is not always significantly larger than $\tau_{c}$, so that the regime in which linear networks can beat the BergPurcell estimate is biologically relevant. For example, both the MAPK response to EGF stimulation [27,28] and the NF- $\kappa$ B response to TNF stimulation [29] peak on the time scale of ligand-receptor debinding (10 min [24] and $30 \mathrm{~min}$ [30], respectively). Additionally, correlation times for gene expression are of the order of the cell cycle time in both $E$. coli and human cells [25,31], suggesting the finite $T_{o}$ limit is also important for scenarios in which intracellular proteins act as receptors for intracellular signals [2].

Interestingly, when $T_{o} \lesssim \tau_{c}$, the equilibration time of the signal must be taken into account, since the equilibration time is, according to the fluctuation-dissipation theorem, given by the correlation time, at least when the change in $c$ is small. Therefore, we end by studying how signaling networks can extract information from nonstationary signals. We study an input signal generated by $\varnothing \rightleftharpoons S$ with $S(0)=0$ and forward and reverse rates $k_{p} c$ and $k_{r} S$, respectively. This signal increases to its steady state value on a time scale $\tau=1 / k_{r}$, which also equals the steadystate correlation time $\tau_{c}$. Extending the procedure in Eqs. (3) and (4), the minimal estimation error with a linear signaling network is $\sigma_{\hat{c}}^{2}\left[X^{*}\left(T_{o}\right)\right]=\frac{\sigma_{\hat{c}}^{2}[S]}{T_{o} /\left(2 \tau_{c}\right)+\ln \left(2-e^{\left.-T_{o} / \tau_{c}\right) / 2}\right.}$ [22]. This shows that less information can be extracted from nonstationary signals than from stationary ones. To avoid the detrimental effect of correlations, the optimal weighting function places more weight on the initial and final points, as for stationary signals. However, because there is no information at $t=0$, the amplification of early time points is spread over time points $t<\tau_{c}$ (Fig. S2 [22]). Additionally, the relative amplification of the last time point increases with decreasing $T_{o}$. Indeed, when $T_{o} \ll \tau_{c}$, no previous signal values are sufficiently uncorrelated with the most recent one, and almost all weight is placed on the final time point $S\left(T_{o}\right)$.

We have studied the ability of linear signaling networks to extract information from noisy input signals. While the data processing inequality suggests that it is advantageous to limit the number of nodes in a signaling network to minimize the effect of intrinsic noise [14], here we show that there can be a competing effect, in terms of information processing, in favor of increasing the number of nodes: better removal of extrinsic noise. Additional nodes make it possible to sculpt the weighting function for averaging the incoming signal, allowing signaling networks to reach and even exceed the Berg-Purcell limit. Our predictions could be tested experimentally in a controlled setting by using in vitro or in vivo synthetic signaling networks [32]. Dual reporter constructs can be used to isolate the effects of extrinsic noise, studied in this Letter, from noise intrinsic to the signaling machinery itself $[33,34]$.

This work is part of the research program of the "Stichting voor Fundamenteel Onderzoek der Materie 
(FOM)", which is financially supported by the "Nederlandse Organisatie voor Wetenschappelijk Onderzoek (NWO)." We thank Andrew Mugler and Wiet de Ronde for a careful reading of the manuscript.

[1] H. C. Berg and E. M. Purcell, Biophys. J. 20, 193 (1977).

[2] W. Bialek and S. Setayeshgar, Proc. Natl. Acad. Sci. U.S.A. 102, 10040 (2005).

[3] K. Wang, W.-J. Rappel, R. Kerr, and H. Levine, Phys. Rev. E 75, 061905 (2007).

[4] W.-J. Rappel and H. Levine, Phys. Rev. Lett. 100, 228101 (2008).

[5] R. G. Endres and N. S. Wingreen, Phys. Rev. Lett. 103, 158101 (2009).

[6] G. Aquino and R. G. Endres, Phys. Rev. E 81, 021909 (2010).

[7] B. Hu, W. Chen, W.-J. Rappel, and H. Levine, Phys. Rev. Lett. 105, 048104 (2010).

[8] T. Mora and N. S. Wingreen, Phys. Rev. Lett. 104, 248101 (2010).

[9] P. J. M. van Haastert and M. Postma, Biophys. J. 93, 1787 (2007).

[10] M. Skoge, Y. Meir, and N. S. Wingreen, Phys. Rev. Lett. 107, 178101 (2011).

[11] R. Heinrich, B. G. Neel, and T. A. Rapoport, Mol. Cell 9, 957 (2002)

[12] E. Ziv, I. Nemenman, and C. H. Wiggins, PLoS ONE 2, e1077 (2007).

[13] S. Tanase-Nicola, P. B. Warren, and P. R. ten Wolde, Phys. Rev. Lett. 97, 068102 (2006).

[14] W. H. de Ronde, F. Tostevin, and P. R. ten Wolde, Phys. Rev. E 82, 031914 (2010).

[15] F. Tostevin and P. R. ten Wolde, Phys. Rev. Lett. 102, 218101 (2009).

[16] G. Tkačik, C. G. Callan, and W. Bialek, Proc. Natl. Acad. Sci. U.S.A. 105, 12265 (2008).

[17] J. Paulsson, Nature (London) 427, 415 (2004).
[18] R. Milo, S. Shen-Orr, S. Itzkovitz, N. Kashtan, D. Chklovskii, and U. Alon, Science 298, 824 (2002).

[19] W.-J. Rappel and H. Levine, Proc. Natl. Acad. Sci. U.S.A. 105, 19270 (2008).

[20] W.H. de Ronde, Ph.D. thesis, Vrije Universiteit, Amsterdam, 2012.

[21] M. Samoilov, A. Arkin, and J. Ross, J. Phys. Chem. A 106, 10205 (2002).

[22] See Supplemental Material at http://link.aps.org/ supplemental/10.1103/PhysRevLett.109.218103 for details about the proof of Eq. (3), multilevel reversible cascades, and measuring concentrations based on nonstationary signals.

[23] X. Gao, P. R. Lowry, X. Zhou, C. Depry, Z. Wei, G. W. Wong, and J. Zhang, Proc. Natl. Acad. Sci. U.S.A. 108, 14509 (2011).

[24] D. A. Lauffenburger and J. Linderman, Receptors (Oxford University Press, Oxford, 1992).

[25] N. Rosenfeld, J. W. Young, U. Alon, P. S. Swain, and M. B. Elowitz, Science 307, 1962 (2005).

[26] S. D. Talia, J. M. Skotheim, J. M. Bean, E. D. Siggia, and F. R. Cross, Nature (London) 448, 947 (2007).

[27] B. Schoeberl, C. Eichler-Jonsson, E. D. Gilles, and G. Muller, Nat. Biotechnol. 20, 370 (2002).

[28] R. Avraham and Y. Yarden, Nat. Rev. Mol. Cell Biol. 12, 104 (2011).

[29] R. Cheong, A. Rhee, C. J. Wang, I. Nemenman, and A. Levchenko, Science 334, 354 (2011).

[30] M. Grell, H. Wajant, G. Zimmerman, and P. Scheurich, Proc. Natl. Acad. Sci. U.S.A. 95, 570 (1998).

[31] A. Sigal, R. Milo, A. Cohen, N. Geva-Zatorsky, Y. Klein, Y. Liron, N. Rosenfeld, T. Danon, N. Perzov, and U. Alon, Nature (London) 444, 643 (2006).

[32] C. J. Bashor, A. A. Horwitz, S. G. Peisajovich, and W. A. Lim, Annu. Rev. Biophys. 39, 515 (2010).

[33] M. B. Elowitz, A. J. Levine, E. D. Siggia, and P. S. Swain, Science 297, 1183 (2002).

[34] C. G. Bowsher and P. S. Swain, Proc. Natl. Acad. Sci. U.S.A. 109, E1320 (2012). 